DESY 94-193

hep-ph/9411241

\title{
Infrared Fixed Points and Fixed Lines in the Top-Bottom-Tau Sector in Supersymmetric Grand Unification *
}

\author{
Barbara Schrempp \\ Universität Kiel, Institut für Theoretische Physik \\ Olshausenstr. 40, D-24098 Kiel \\ and \\ Deutsches Elektronen-Synchrotron DESY \\ Notkestr.85, D-22603 Hamburg
}

October 1994

\begin{abstract}
The two-loop "top-down" renormalization group flow for the top, bottom and tau Yukawa couplings, from $\mu=M_{\mathrm{GUT}} \simeq O\left(10^{16} \mathrm{GeV}\right)$ to $\mu \simeq m_{t}$, is explored in the framework of supersymmetric grand unification; reproduction of the physical bottom and tau masses is required. Instead of following the recent trend of implementing exact Yukawa coupling unification i) a search for infrared (IR) fixed lines and fixed points in the $m_{t}^{\text {pole }}$ $\tan \beta$ plane is performed and ii) the extent to which these imply approximate Yukawa unification is determined. In the $m_{t}^{\text {pole }}-\tan \beta$ plane two IR fixed lines, intersecting in an IR fixed point, are located. The more attractive fixed line has a branch of almost constant top mass, $m_{t}^{\text {pole }} \simeq 168-180 \mathrm{GeV}$ (close to the experimental value), for the large interval $2.5 \lesssim \tan \beta \lesssim 55$; it realizes tau-bottom Yukawa unification at $M_{\mathrm{GUT}}$ approximately. The less attractive fixed line as well as the fixed point at $m_{t}^{\text {pole }} \simeq 170 \mathrm{GeV}, \tan \beta \simeq 55$ implement approximate top-bottom Yukawa unification at all scales $\mu$. The renormalization group flow is attracted towards the IR fixed point by way of the more attractive IR fixed line. The fixed point and lines are distinct from the much quoted effective IR fixed point $m_{t}^{\text {pole }} \simeq O(200 \mathrm{GeV}) \sin \beta$.
\end{abstract}

* project supported in part by Deutsche Forschungsgemeinschaft 
There has been renewed interest in supersymmetric grand unification triggered by the recent sucessful reevaluation of gauge coupling unification [1]. Also the appealing concepts of taubottom Yukawa coupling unification, e.g. valid [2] in a minimal $S U(5)$ theory, or possibly even of tau-bottom-top Yukawa unification have been revived [3]- [6]. The main issue has been to determine how exact tau-bottom (-top) Yukawa coupling unification at the unification scale $\mu=M_{\mathrm{GUT}} \simeq O\left(10^{16} \mathrm{GeV}\right)$ constrains the low energy parameters of the top-bottom-tau sector at the infrared (IR) scale $\mu=m_{t}$. In this paper a reversed strategy is followed. First the unconstrained "top-down" renormalization group (RG) flow in the top-bottom-tau sector, from $\mu=M_{\mathrm{GUT}}$ to $\mu=m_{t}$, is explored. The aim is, to determine the IR fixed lines and IR fixed points in the low energy top-bottom-tau sector, which attract the RG flow. Then the extent to which these distinguished IR lines and points reflect approximate Yukawa coupling unification at $M_{\mathrm{GUT}}$ is investigated.

The simplest framework is the minimal supersymmetric extension of the Standard Model (MSSM), embedded in a "one-step" grand unified theory (e.g. an $S U(5)$ theory). It holds from $M_{\mathrm{GUT}}$ down to a scale $\mu=M_{\mathrm{SUSY}}$ of the order of the weak interaction scale, parametrizing effectively the thresholds of the supersymmetric particles and the heavy Higgs bosons. For simplicity $M_{\mathrm{SUSY}}=m_{t}$ is chosen in the following. The top-bottom-tau sector is described by the "top down" RG evolution of the Yukawa couplings $h_{t}(\mu), h_{b}(\mu), h_{\tau}(\mu)$, from $\mu=M_{\mathrm{GUT}}$ to $\mu=m_{t}$. The running top, bottom and tau masses at $\mu=m_{t}$ are

$$
\begin{aligned}
& m_{t}\left(m_{t}\right)=h_{t}\left(m_{t}\right) \sin \beta v / \sqrt{2}, \\
& m_{b}\left(m_{t}\right)=h_{b}\left(m_{t}\right) \cos \beta v / \sqrt{2}, \\
& m_{\tau}\left(m_{t}\right)=h_{\tau}\left(m_{t}\right) \cos \beta v / \sqrt{2} .
\end{aligned}
$$

Here $\tan \beta=v_{2} / v_{1}$ parametrizes the unknown ratio of the vacuum expectation values $v_{1}, v_{2}$ of the neutral components of the two Higgs doublets, with $v_{1}^{2}+v_{2}^{2}=v^{2} \simeq(246 \mathrm{GeV})^{2}$. The physical top mass is given by the pole mass [7]

$$
m_{t}^{\text {pole }}=m_{t}\left(m_{t}\right)\left(1+\frac{4}{3 \pi} \alpha_{3}\left(m_{t}\right)+O\left(\alpha_{3}^{2}\right)\right) .
$$

In a "top-down" RG analysis of the top-bottom-tau sector there are four free parameters to start with, $\tan \beta$ and e.g. $h_{t 0}, h_{b 0}, h_{\tau 0}$, the ultraviolet (UV) initial values of the Yukawa couplings at $\mu=M_{\mathrm{GUT}}$. Two IR conditions are provided by the physical masses $m_{\tau}, m_{b}$ (evolved to $\mu=m_{t}$ within the Standard Model). This leaves e.g. $\tan \beta$ and the IR parameter $m_{t}^{\text {pole }}$ free.

Let me briefly recapitulate the consequences [3]- [6] of implementing exact tau-bottom Yukawa coupling unification, $h_{\tau 0}=h_{b 0}$, at $M_{\mathrm{GUT}}$. The allowed $\tan \beta, m_{t}^{\text {pole }}$ values are constrained to a narrow band in the $m_{t}^{\text {pole }}-\tan \beta$ plane, which turns out [3]- [6] surprisingly to coincide essentially with the triviality bound determined by the IR image of large UV initial values $h_{t 0}$, the much discussed strongly attractive IR fixed point [8]-14]

$$
m_{t}^{\text {pole }} \simeq O(200 \mathrm{GeV}) \sin \beta .
$$

Compared with the direct [18] and indirect [19] experimental evidences for the top mass

$$
\text { direct evidence } m_{t}=174 \pm 10{ }_{-12}^{+13} \mathrm{GeV} \text {, indirect evidence } m_{t}=173 \begin{gathered}
+12+18 \\
-13-20
\end{gathered} \mathrm{GeV} \text {, }
$$

\footnotetext{
${ }^{1}$ Usually also Refs. 15, 16, 17] are quoted along with Ref. [8] in this context; however, it will be important in the following that they refer to a different though nearby fixed point.
} 
most likely only two narrow windows, one at small $\tan \beta \simeq O(1.5)$ and one at large $\tan \beta \simeq$ $O(60)$ are admitted. Top-bottom-tau Yukawa unification is realized near $\tan \beta \simeq 60$.

For the following it is important to realize that the fixed point (5) is rather what has been called [8] an effective fixed point, since neither does its position remain fixed under the "top down" evolution nor does it attract RG solutions from below.

In this paper the two-loop RG flow in $h_{t}(\mu), h_{b}(\mu), h_{\tau}(\mu)$ for the "top down" evolution, from $M_{\mathrm{GUT}}$ to $m_{t}$, is investigated. This implies studying solutions starting their evolution at arbitrary initial values $h_{t 0}, h_{b 0}, h_{\tau 0}$ within the perturbatively allowed range $h_{t 0}^{2} / 4 \pi, h_{b 0}^{2} / 4 \pi$, $h_{\tau 0}^{2} / 4 \pi<O(1)$ and for an arbitrary value of $\tan \beta$, only subject to the condition that the physical masses $m_{b}$ and $m_{\tau}$ be reproduced. No Yukawa coupling unification is imposed; so at $\mu=m_{t}$ essentially the whole $m_{t}^{\text {pole }}-\tan \beta$ plane, bounded from above by the triviality bound (the IR images of large $h_{t 0}$ or $h_{b 0}$ ), is available for investigation. The main issues are then

- to search for genuine IR fixed points and fixed lines in the $m_{t}^{\text {pole }}$ - $\tan \beta$ plane, which attract the RG flow from above as well as from below,

- to determine the strength of their IR attraction and thus a hierarchy of their importance,

- to investigate to which extent Yukawa coupling unification can be maintained on an approximate level.

Let me briefly anticipate the most interesting resulting IR fixed manifolds in the $m_{t}^{\text {pole }}$ $\tan \beta$ plane, the IR fixed line $m_{t}^{\text {pole }} \simeq(170-180) \mathrm{GEV} \sin \beta$ as well as the IR fixed point $m_{t}^{\text {pole }} \simeq$ $170 \mathrm{GeV}, \tan \beta \simeq 55$, both implying an almost constant top mass $m_{t}^{\text {pole }} \simeq 168-180 \mathrm{GeV}$ for the large interval $2.5 \lesssim \tan \beta \lesssim 55$. This top mass is in good agreement with the experimental top mass value (6). The issue of top-bottom Yukawa coupling unification will enter the discussion automatically, since one IR fixed line as well as the IR fixed point imply it approximately for all scales $\mu$. Tau-bottom Yukawa unification at $M_{\mathrm{GUT}}$ will turn out to hold only approximately.

Let me mention in this context that IR fixed lines are of particular interest, if they are more strongly attractive than IR fixed points. This happens e.g. in the Standard Model, where an IR fixed line, corresponding to a top-Higgs mass relation, has turned out [17 to be much more strongly attractive than the corresponding IR fixed point [15, 16, 17]. An extended analysis of the Higgs-top-bottom-tau sector in the Standard Model is in preparation [20.

Let me point out that what physicists like to call a "fixed line" is termed in mathematical language [21] more appropriately an "invariant line"; this terminology has been used in Ref. [17.

The two-loop RG equations [22, 3] of the top-bottom-tau sector of the MSSM involve $g_{3}, g_{2}$, $g_{1}$, the $S U(3) \times S U(2) \times U(1)$ gauge couplings, and the Yukawa couplings $h_{t}, h_{b}, h_{\tau}$ running as functions of $t=\ln \left(\mu / M_{\mathrm{GUT}}\right)$, assuming vanishing $\mathrm{u}, \mathrm{d}, \mathrm{s}, \mathrm{c}, \mathrm{e}, \mu$ Yukawa couplings. Before turning to the numerical two-loop RG analysis, considerable analytical insight into the IR fixed manifolds will be obtained from the corresponding one-loop RG equations with vanishing electroweak gauge couplings, $g_{1}, g_{2}=0$. In this reduced framework, which is a good approximation except for the small region in coupling space, where $g_{1}, g_{2}$ are not dominated by any of the other couplings, the IR fixed points and fixed lines are exact. They will appear in the variables

$$
\rho_{t}=\frac{h_{t}^{2}}{g_{3}^{2}}, \quad \rho_{b}=\frac{h_{b}^{2}}{g_{3}^{2}}, \quad \rho_{\tau}=\frac{h_{\tau}^{2}}{g_{3}^{2}},
$$

and not in $h_{t}, h_{b}, h_{\tau}$. By eliminating the variable $t$ in favour of $g_{3}^{2}$ the top-bottom-tau sector in the reduced one -loop framework is described by the following RG equations in terms of the 
three variables (7)

$$
\begin{aligned}
-3 g_{3}^{2} \frac{\mathrm{d} \rho_{t}}{\mathrm{~d} g_{3}^{2}} & =\rho_{t}\left(6 \rho_{t}+\rho_{b}-\frac{7}{3}\right), \\
-3 g_{3}^{2} \frac{\mathrm{d} \rho_{b}}{\mathrm{~d} g_{3}^{2}} & =\rho_{b}\left(6 \rho_{b}+\rho_{t}+\rho_{\tau}-\frac{7}{3}\right), \\
-3 g_{3}^{2} \frac{\mathrm{d} \rho_{\tau}}{\mathrm{d} g_{3}^{2}} & =\rho_{\tau}\left(3 \rho_{b}+4 \rho_{\tau}+3\right) .
\end{aligned}
$$

Clearly, this system of coupled differential equations has four (finite) fixed points, of which only the fourth one (14) is IR attractive

$$
\begin{gathered}
\rho_{t}=0 \text { (IR repulsive), } \rho_{b}=0 \text { (IR repulsive), } \rho_{\tau}=0 \text { (IR attractive), } \\
\rho_{t}=\frac{7}{18} \text { (IR attractive), } \rho_{b}=0 \text { (IR repulsive), } \rho_{\tau}=0 \text { (IR attractive), } \\
\rho_{t}=0 \text { (IR repulsive), } \rho_{b}=\frac{7}{18} \text { (IR attractive) } \rho_{\tau}=0 \text { (IR attractive), } \\
\rho_{t}=\frac{1}{3} \text { (IR attractive), } \rho_{b}=\frac{1}{3} \text { (IR attractive) }, \rho_{\tau}=0 \text { (IR attractive). }
\end{gathered}
$$

The fixed point (12) is the supersymmetric counterpart of a well-known fixed point in the Standard Model [15, 16, 17]. It is, however, much more IR attractive with respect to $\rho_{t}$, since it is approached as

$$
\rho_{t}\left(g_{3}^{2}\right)=\frac{7 / 18}{1-\left(1-\frac{7}{18 \rho_{t 0}}\right)\left(\frac{g_{3}^{2}}{g_{30}^{2}}\right)^{-7 / 9}}
$$

for $\rho_{b, \tau}=0$. The measure of the IR attraction is the size of the negative power in $\left(g_{3}^{2} / g_{30}^{2}\right)^{-7 / 9}$, i.e. $-7 / 9$, to be compared with the corresponding one, $-1 / 7$, in the Standard Model. The discussion of the fixed point (13) runs analogously, with the roles of $\rho_{t}$ and $\rho_{b}$ interchanged.

Linearization of the system of differential equations (8)-(10) in the neighbourhood of the genuine IR fixed point (14) leads to the following approximate analytical solution

$$
\begin{aligned}
\rho_{t}\left(g_{3}^{2}\right) \simeq & \frac{1}{3}+\frac{1}{2}\left(\left(\rho_{t 0}+\rho_{b 0}-\frac{2}{3}-\frac{1}{5} \rho_{\tau 0}\right)\left(\frac{g_{3}^{2}}{g_{30}^{2}}\right)^{-7 / 9}+\left(\rho_{t 0}-\rho_{b 0}+\frac{1}{7} \rho_{\tau 0}\right)\left(\frac{g_{3}^{2}}{g_{30}^{2}}\right)^{-5 / 9}\right. \\
& \left.+\frac{2}{35} \rho_{\tau 0}\left(\frac{g_{3}^{2}}{g_{30}^{2}}\right)^{-4 / 3}\right), \\
\rho_{b}\left(g_{3}^{2}\right) \simeq & \frac{1}{3}+\frac{1}{2}\left(\left(\rho_{t 0}+\rho_{b 0}-\frac{2}{3}-\frac{1}{5} \rho_{\tau 0}\right)\left(\frac{g_{3}^{2}}{g_{30}^{2}}\right)^{-7 / 9}-\left(\rho_{t 0}-\rho_{b 0}+\frac{1}{7} \rho_{\tau 0}\right)\left(\frac{g_{3}^{2}}{g_{30}^{2}}\right)^{-5 / 9}\right. \\
& \left.+\frac{12}{35} \rho_{\tau 0}\left(\frac{g_{3}^{2}}{g_{30}^{2}}\right)^{-4 / 3}\right), \\
\rho_{\tau}\left(g_{3}^{2}\right) \simeq & \rho_{\tau 0}\left(\frac{g_{3}^{2}}{g_{30}^{2}}\right)^{-4 / 3},
\end{aligned}
$$

where $\rho_{t 0}, \rho_{b 0}, \rho_{\tau 0}, g_{30}^{2}$ are the corresponding initial values at the UV scale.

Mathematical theorems [21] about systems of coupled differential equations with several fixed points imply that the system (8)-(10) has an IR attractive fixed plane (invariant plane), the $\rho_{t}-\rho_{b}$ plane, $\rho_{\tau}\left(g_{3}^{2}\right) \equiv 0$, and in this plane two IR fixed lines (invariant lines) intersecting in the IR fixed point (14), $\rho_{t}=\rho_{b}=1 / 3$; the two fixed lines are 
- $\rho_{t}\left(g_{3}^{2}\right) \equiv \rho_{b}\left(g_{3}^{2}\right)$, equivalent to $h_{t}\left(g_{3}^{2}\right) \equiv h_{b}\left(g_{3}^{2}\right)$, implying exact top-bottom Yukawa coupling unification at all scales $\mu$,

- the solution of Eqs. (8), (9) for $\rho_{\tau}=0$ which interpolates the three fixed points (12)-(14), i.e. $\left(\rho_{t}=7 / 18, \rho_{b}=0\right),\left(\rho_{t}=1 / 3, \rho_{b}=1 / 3\right),\left(\rho_{t}=0, \rho_{b}=7 / 18\right)$, having roughly the shape of two adjacent sides of a square.

The first fixed line can be read off from

$$
-3 g_{3}^{2} \frac{\mathrm{d}\left(\frac{\rho_{b}}{\rho_{t}}\right)}{\mathrm{d} g_{3}^{2}}=5 \rho_{b}\left(\frac{\rho_{b}}{\rho_{t}}-1\right),
$$

as obtained from Eqs. (8), (9) for $\rho_{\tau}=0$. As may be inferred from Eqs. (15)-(18), the fixed $\rho_{t}-\rho_{b}$ plane, $\rho_{\tau} \equiv 0$, is IR attractive like $\left(g_{3}^{2} / g_{30}^{2}\right)^{-4 / 3}$ near the fixed point (14), the square-type fixed line is the more strongly attractive one, attracting like $\left(g_{3}^{2} / g_{30}^{2}\right)^{-7 / 9}$ in its neighbourhood, the $\rho_{t}=\rho_{b}$ fixed line is the less attractive one, attracting like $\left(g_{3}^{2} / g_{30}^{2}\right)^{-5 / 9}$ near the IR fixed point (14).

Altogether, the "top-down" RG flow is attracted towards the IR fixed point (14) by way of first being attracted towards the square-type IR fixed line in the $\rho_{t}-\rho_{b}$ plane and then (practically) along this line towards the fixed point. Of course, solutions with initial values on an IR fixed line are drawn along it towards the IR fixed point.

Reinstating the electroweak couplings $g_{1}, g_{2}$ and using the full two-loop RG equations [22, 3], the fixed points and fixed lines are found not to be exact any more. In order to determine the approximate positions of the fixed points and fixed lines one could follow Ref. [15 and replace $g_{1}, g_{2}$ by constants, given by their averages along the evolution path. This introduces a weak dependence on the evolution path, in particular on the UV scale. Here, it is preferred to maintain properly running couplings $g_{1}(\mu), g_{2}(\mu)$ and to follow a prescription, which has already been successfully applied in Ref. [17]. For a given evolution path, e.g. from $\mu=M_{\mathrm{GUT}}$ to $\mu=m_{t}$,

- the approximate IR fixed point in the $\rho_{t}-\rho_{b}$ plane is determined as the unique point which has the same value at $\mu=M_{\mathrm{GUT}}$ and at $\mu=m_{t}$,

- an approximate IR fixed line in the $\rho_{t}-\rho_{b}$ plane is determined by the condition that all points, which start on it or - in case of the most attractive line - sufficiently close to it at $\mu=M_{\mathrm{GUT}}$, end on it at $\mu=m_{t}$.

Again the results depend somewhat on the evolution path, in particular on the UV scale; the numerical deviations from the positions given in Eqs. (12)-(14) are the larger, the larger the UV scale.

Obviously, the scenario of grand unification is not really vital for the results. The IR fixed lines and fixed point are basically IR properties of the MSSM. Only to the extent that they are not exact but approximate do they depend on the evolution path, i.e. on the size of $M_{\mathrm{GUT}}$.

The following numerical RG analysis uses for $\mu \leq m_{t}$ the same Standard Model evolutions for $g_{1}, g_{2}, g_{3}, m_{\tau}, m_{b}$ as Ref. [3], comprising three-loop expressions for $\alpha_{3}$ and $m_{b}$, with slightly different actualized input parameters. For $\mu \geq m_{t}$ the two-loop RG evolution equation of the MSSM [22, 3] are used for vanishing first and second generation Yukawa couplings. No error analysis is performed. The inputs are as follows

- The supersymmetry scale is chosen at $M_{\mathrm{SUSY}}=m_{t}=174 \mathrm{GeV}$. 
- From Ref. 23 $m_{Z}=91.187 \mathrm{GeV}, \sin ^{2} \hat{\theta}_{Z}\left(m_{Z}\right)=0.2319, \alpha_{\mathrm{em}}\left(m_{Z}\right)=127.9$ leading to $g_{1}^{2}\left(m_{Z}\right)=0.2132, g_{2}^{2}\left(m_{Z}\right)=0.4237$.

- The grand unification scale $M_{\mathrm{GUT}}$ as well as $\alpha_{3}\left(m_{Z}\right)$ are determined by requiring unification of all three gauge couplings at $\mu=M_{\mathrm{GUT}}$ (neglecting the two-loop Yukawa coupling contributions in the gauge coupling RG equations, following Ref. [3]). The results are $M_{\mathrm{GUT}}=2.22310^{16} \mathrm{GeV}, g_{1}^{2}=g_{2}^{2}=g_{3}^{2}=0.5278$ at $M_{\mathrm{GUT}}$ and $\alpha_{3}\left(m_{Z}\right)=0.1247$.

$$
m_{\tau}=1.7771 \mathrm{GeV}[23] \text { and } m_{b}=4.25 \mathrm{GeV} \text { [24], }
$$

are used, admitting a variation within $4.1 \mathrm{GeV} \leq m_{b} \leq 4.4 \mathrm{GeV}$ [3]. The evolutions to $\mu=m_{t}$ are evaluated to be given by

$$
m_{b}\left(m_{t}\right)=m_{b} / \eta_{b}, \quad m_{\tau}\left(m_{t}\right)=m_{\tau} / \eta_{\tau} \text { with } \eta_{b}=1.599, \quad \eta_{\tau}=1.02
$$

- RG evolutions are performed from $\mu=M_{\mathrm{GUT}}$ to $\mu=m_{t}=174 \mathrm{GeV}$.

Of primary interest is to investigate the RG flow in the variables $\rho_{t}=h_{t}^{2} / g_{3}^{2}, \rho_{b}=h_{b}^{2} / g_{3}^{2}$, $\rho_{\tau}=h_{\tau}^{2} / g_{3}^{2}$ from $M_{\mathrm{GUT}}$ to $m_{t}$ (subject to the condition that the physical tau and bottom masses be reproduced), to locate the IR fixed point and fixed lines in the $\rho_{t}-\rho_{b}$ plane, and to display their respective strenghts of attraction.

This is achieved in Fig. 1 as follows: 36 pairs of initial values $\left(\rho_{t 0}, \rho_{b 0}\right)$ are chosen along the boundary of a square, defined by its four corners $(0.0001,0.0001),(0.0001,25),(25,25)$, $(25,0.0001)$; the value 25 is the limit of validity of perturbation theory. The evolution from $\mu=M_{\mathrm{GUT}}$ to $\mu=m_{t}$ maps the 36 initial UV points onto 36 final IR points, which are the starting points of 36 polygons in Fig. 1 . The 36 IR points are taken as a new set of UV initial values, evolved to a new set of IR final points. This procedure is repeated until all final points meet in the IR fixed point. This leads to 36 sequences of evolutions. In order to guide the eye, the IR points of each sequence are connected by straight lines to form the 36 polygons displayed in Fig. 1.

For each of these evolutions the corresponding pair of initial values $\left(\rho_{t 0}, \rho_{b 0}\right)$ is complemented by a $\rho_{\tau 0}$ value, which is adjusted such that after the evolution the appropriate ratio $\rho_{\tau}\left(m_{t}\right) / \rho_{b}\left(m_{t}\right)=m_{\tau}^{2}\left(m_{t}\right) / m_{b}^{2}\left(m_{t}\right)$ at $\mu=m_{t}$ is reproduced, which is fixed by Eqs. (20) and(21). This requires values for $\sqrt{\rho_{\tau 0} / \rho_{b 0}}=h_{\tau 0} / h_{b 0}$ ranging between 0.92 and 2.1 .

The paths which the polygons take in the $\rho_{t}-\rho_{b}$ plane in Fig. 1 demonstrate that there is a rather strongly IR attractive fixed line. It has two branches, labelled by " 1 " and " 2 " in Fig. 1. Branch " 1 " has almost constant $\rho_{t}$ and is of physical interest; it runs from $\rho_{t}=0.689$, $\rho_{b}=0$ (the analogon of the fixed point (12) and the supersymmetric counterpart of the fixed point in Refs. [15, 16, 17]) to the IR fixed point at $\rho_{t}=.609, \rho_{b}=.505$; branch "2", being almost constant in $\rho_{b}$, runs from $\rho_{t}=0, \rho_{b}=0.586$ (the analogon of the fixed point (13)) to the fixed point. A less attractice IR fixed line, labelled by " 3 " in Fig. 1, is seen to be $\rho_{t} \simeq 1.2 \rho_{b}$, intersecting the more attractive IR fixed line in the IR fixed point. This line corresponds to $h_{t} \simeq 1.1 h_{b}$, implying approximate top-bottom Yukawa coupling unification at all scales $\mu$. The IR fixed point as well as the two IR fixed lines are as expected from the preceding analytical considerations only with shifted positions due to the effect of including the electroweak gauge couplings and to a lesser extent to the two-loop contributions. The lengths of the sections of the polygons are a direct measure of the strength of IR attraction: the longer the section, the stronger the attraction. Clearly, as expected, the RG flow is primarily attracted towards the 
more attractive IR fixed line and then runs (practically) along it towards the IR fixed point. The IR final points coming from UV initial points with $\rho_{t 0}=25$ or $\rho_{b 0}=25$ are connected by a dashed line; this line represents the triviality bound. In the neighbourhood of branch " 1 " as well as near the IR fixed point, a ratio $\sqrt{\rho_{\tau 0} / \rho_{b 0}}=h_{\tau 0} / h_{b 0} \simeq 1.3$ is required, which realizes tau-bottom Yukawa coupling unification at $M_{\mathrm{GUT}}$ only approximately.

Fig. 1 in terms of the variables $\rho_{t}=h_{t}^{2} / g_{3}^{2}$ and $\rho_{b}=h_{b}^{2} / g_{3}^{2}$ is translated] into Fig. 2 for the variables $m_{t}^{\text {pole }}$ and $\tan \beta$ by means of Eqs. (11), (2), (41), (20) and (21) and by enforcing $m_{b}=4.25 \mathrm{GeV}$. The maps of the infrared fixed lines are again labelled consistently by "1", "2" and " 3 ". The dotted line is again the triviality bound, one branch of which is the effective IR fixed point (5)

The more strongly attractive IR fixed line, branch "1", is not sufficiently attractive to draw all perturbatively allowed initial values $h_{t 0}<3.6$ onto it, given an RG evolution from $M_{\mathrm{GUT}}$ to $m_{t}$. However, it is remarkable that all initial values $0.6 \lesssim h_{t 0}<3.6$ are drawn from above and from below within a strip corresponding to $m_{t}^{\text {pole }} \simeq 174 \pm 23 \mathrm{GeV} \sin \beta$. The IR fixed point is less attractive.

Fig 3 shows the dependence of the results on $m_{b}$ : for the values $m_{b}=4.1,4.25,4.4 \mathrm{GeV}$ the pairs of IR fixed lines intersecting in their common IR fixed points $m_{t}^{\text {pole }}=169.6 \mathrm{GeV}$, $\tan \beta=57.4,55.4,53.5$, respectively, are displayed. Clearly, the physically interesting branch "1" is hardly affected by this variation.

The rich IR fixed manifolds, displayed in Figs. 1 and 2, are summarized according to their manifestations in the $h_{t}-h_{b}$ plane resp. in the $m_{t}^{\text {pole }}-\tan \beta$ plane

- a more strongly attractive IR fixed line (invariant line) with the two branches

i) branch "1":

$$
h_{t} \simeq .93-.99 \text { resp. } m_{t}^{\text {pole }} \simeq(170-180) \mathrm{GeV} \sin \beta,
$$

implying an almost constant top mass $m_{t}^{\text {pole }} \simeq 168-180 \mathrm{GeV}$ for the large interval $2.5 \lesssim \tan \beta \lesssim 55$; in its neighborhood approximate tau-bottom Yukawa coupling unification at $\mu=M_{\mathrm{GUT}}$ is realized

$$
h_{\tau 0} \simeq 1.3 h_{b 0} .
$$

ii) branch "2":

$$
h_{b} \simeq .85-.91 \text { resp. } \tan \beta \simeq 55-60,
$$

- a less strongly attractive IR fixed line (invariant line), denoted by "3",

$$
h_{t} \simeq 1.1 h_{b} \text { resp. } \quad m_{t}^{\text {pole }} \simeq 3 \mathrm{GeV} \tan \beta,
$$

implying approximate top-bottom Yukawa coupling unification at all scales $\mu$,

- an attractive IR fixed point at their intersection

$$
h_{t} \simeq 0.93, h_{b} \simeq 0.85 \text { resp. } \quad m_{t}^{\text {pole }} \simeq 170 \mathrm{GeV}, \tan \beta \simeq 55,
$$

implying both, approximate top-bottom Yukawa unification at all scales $\mu, h_{t} \simeq 1.1 h_{b}$, as well as approximate tau bottom Yukawa unification, $h_{\tau 0} \simeq 1.3 h_{b 0}$, at $\mu=M_{\mathrm{GUT}}$.

\footnotetext{
${ }^{2}$ by translating the IR points of Fig. 1 and then connecting them again by polygons
} 
The "top-down" RG flow is attracted towards the IR fixed point by way of the more attractive IR fixed line.

From this list a physically appealing solution can be extracted with the following properties:

- it is part of the most attractive IR fixed line ending in the IR fixed point, which both attract the "top-down" RG flow from above and from below,

- it corresponds to an almost constant top mass value, $m_{t}^{\text {pole }} \simeq 168-180 \mathrm{GeV}$, matching the experimental top mass,

- it holds for the large range $2.5 \lesssim \tan \beta \lesssim 55$,

- it implies approximate tau-bottom Yukawa unification at $M_{\mathrm{GUT}}$, at the level $h_{\tau 0} \simeq 1.3 h_{b 0}$,

- for $\tan \beta \simeq 55$ it fulfills in addition approximate top-bottom Yukawa unification at all scales $\mu$, at the level $h_{t} \simeq 1.1 h_{b}$.

Let me emphasize that the constant top mass value of $168-180 \mathrm{GeV}$ as well as the tau-bottom (-top) Yukawa coupling unification, even though valid only approximately, are encoded in the positions of the IR fixed manifolds (22)-(26) and are not put in by hand at any stage.

The solution described above is rather dominated by IR aspects. It has to be contrasted with the somewhat complementary solution [3]- [6] discussed in the introduction which is rather dominated by UV aspects : implementation of exact tau-bottom Yukawa unification, $h_{\tau 0}=h_{b 0}$, at $M_{\mathrm{GUT}}$, which drives the low energy solution towards the triviality bound, requiring large initial UV values $h_{t 0}$, and admitting only two $\tan \beta$ windows, $\tan \beta \simeq O(1.5)$ and $\tan \beta \simeq O(60)$.

It would be interesting to investigate whether threshold effects and the influence of higher dimensional operators at $M_{\mathrm{GUT}}$, which have been considered [25] as possible sources for slight violations of exact gauge coupling unification, could be responsible for violations of exact Yukawa coupling unification to the extent implied in Eqs. (23) and (25).

Of course all numerical results depend to some extent on the input parameters chosen, in particular on the position of $M_{\mathrm{SUSY}}$ and the value of $\alpha_{3}\left(m_{Z}\right)$. A more extended analysis, varying these parameters, is in progress; as expected, no major changes in the resulting IR fixed structures in the $m_{t}^{\text {pole }}-\tan \beta$ plane are found.

Acknowledgements: I thank F. Schrempp for constructive comments after reading the manuscript. I also thank V. Barger and M.S. Berger for a helpful communication. I am grateful to the DESY Theory Group for the extended hospitality.

\section{References}

[1] U. Amaldi, W. de Boer and H. Fürstenau, Phys. Lett B260 (1991) 447; J. Ellis, S. Kelley and D.V. Nanopoulos, Phys. Lett B249 (1990) 441; P. Langacker and M. Luo, Phys. Rev. D44, (1991) 817; F. Anselmo, L. Cifarelli, A. Pe

terman and A. Zichichi, Nuovo Cimento 104A (1991) 1817.

[2] M.S. Chanowitz, J. Ellis and M.K. Gaillard, Nucl. Phys. B128 (1977) 506; A.J. Buras, J. Ellis, M.K. Gaillard and D.V. Nanopoulos, ibid. B135 (1978) 66.

[3] V. Barger, M.S. Berger and P. Ohmann, Phys Rev. D47 (1993) 1039. 
[4] G. Anderson, S. Dimopoulos, L.J. Hall and S. Raby, Phys. Rev. D47 (1993) 3072;

[5] M. Carena, S. Pokorski and C.E.M. Wagner, Nucl. Phys. B406 (1993) 59; W. Bardeen, M. Carena, S. Pokorski and C.E.M. Wagner, Phys. Lett 320 (1994) 110.

[6] P. Langacker and N. Polonsky, Phys. Rev. D49 (1994) 1454.

[7] R. Tarrach, Nucl. Phys. B183 (1981) 384.

[8] C.T. Hill, Phys. Rev. D24 (1981) 691.

[9] J. Bagger, S. Dimopoulos and E. Masso, Phys. Rev Lett. 55 (1985) 1450;

[10] C.D. Frogatt, I.G. Knowles and R.G. Moorhouse, Phys. Lett B249 (1990) 273; ibid. B298 (1993) 356 .

[11] S. Dimopoulos, L. Hall and S. Raby, Phys. Rev. Lett. 68 (1992) 1984; Phys. Rev. D45 (1992) 4192.

[12] V. Barger, M.S. Berger, T. Han, M. Zralek, Phys. Rev. Lett. 68 (1992) 3394; V. Barger, M.S. Berger, P. Ohmann and R.J.N. Phillips, Phy. Lett. B314 (1993) 351; V. Barger, M.S. Berger and P. Ohmann, HEPPH-9409342, Talk given at 2 7th International Conference on High Energy Physics, Glasgow (1994).

[13] W.A. Bardeen, M. Carena, T.E. Clark, K. Sasaki and C.E.M. Wagner, Nucl. Phys. B369 (1992) 33.

[14] B.C. Allanach and S.F. King, Phys. Lett. B328 (1994) 360.

[15] B. Pendleton and G.G. Ross, Phys. Lett. 98B (1981) 291.

[16] W. Zimmermann, Commun. Math. Phys. 97 (1985) 211; J. Kubo, K. Sibold and W. Zimmermann, Nucl. Phys. B259 (1985) 331; Phys. Lett. B220 (1989) 185.

[17] B. Schrempp and F. Schrempp, Phys. Lett. B299 (1993) 321.

[18] CDF collaboration, F. Abe et al., Phys. Rev. Lett. 73 (1994) 225; Phys. Revi D50 (1994) 2966.

[19] LEP electroweak working group, rapporteur talk D. Schaile, to be published in Proc. of the Int. Conf. on High Energy Physiscs, Glasgow (1994).

[20] B. Schrempp, F. Schrempp and M. Wimmer, to be published.

[21] see e.g. J. Guckenheimer and P. Holmes, Non-linear Oscillations, Dynamical Systems and Bifurcations of Vector Fields, Appl. Math. Sciences, Vol. 42, Springer Verlag (1983).

[22] K. Inoue, A. Kakuto, H. Komatsu and S. Takeshita, Prog. Theor. Phys. 67 (1982) 1889; D.R.T. Jones and L. Mezincescu, Phys Lett. 136B (1984) 242; P. West, ibid. 137B (1984) 371; A. Parkes und P. West, ibid. 138B (1984)

99; D.R.T. Jones and L Mezincescu, ibid. 138B (1984) 293; J.E. Björkman and D.R.T. Jones, Nucl. Phys. B259 (1985) 533. 
[23] Particle Data Group, L. Montanet et al., Phys. Rev. D50 (1994) 1173.

[24] J. Gasser and H. Leutwyler, Phys. Rep. 87 (1982) 77.

[25] P. Langacker and N. Polonsky, Phys. Rev. D47 (1993) 4028; R. Barbieri and L.J. Hall, Phys. Rev. Lett. 68 (1992) 752; L.J. Hall and U. Sarid, ibid. 70 (1993) 2673. 


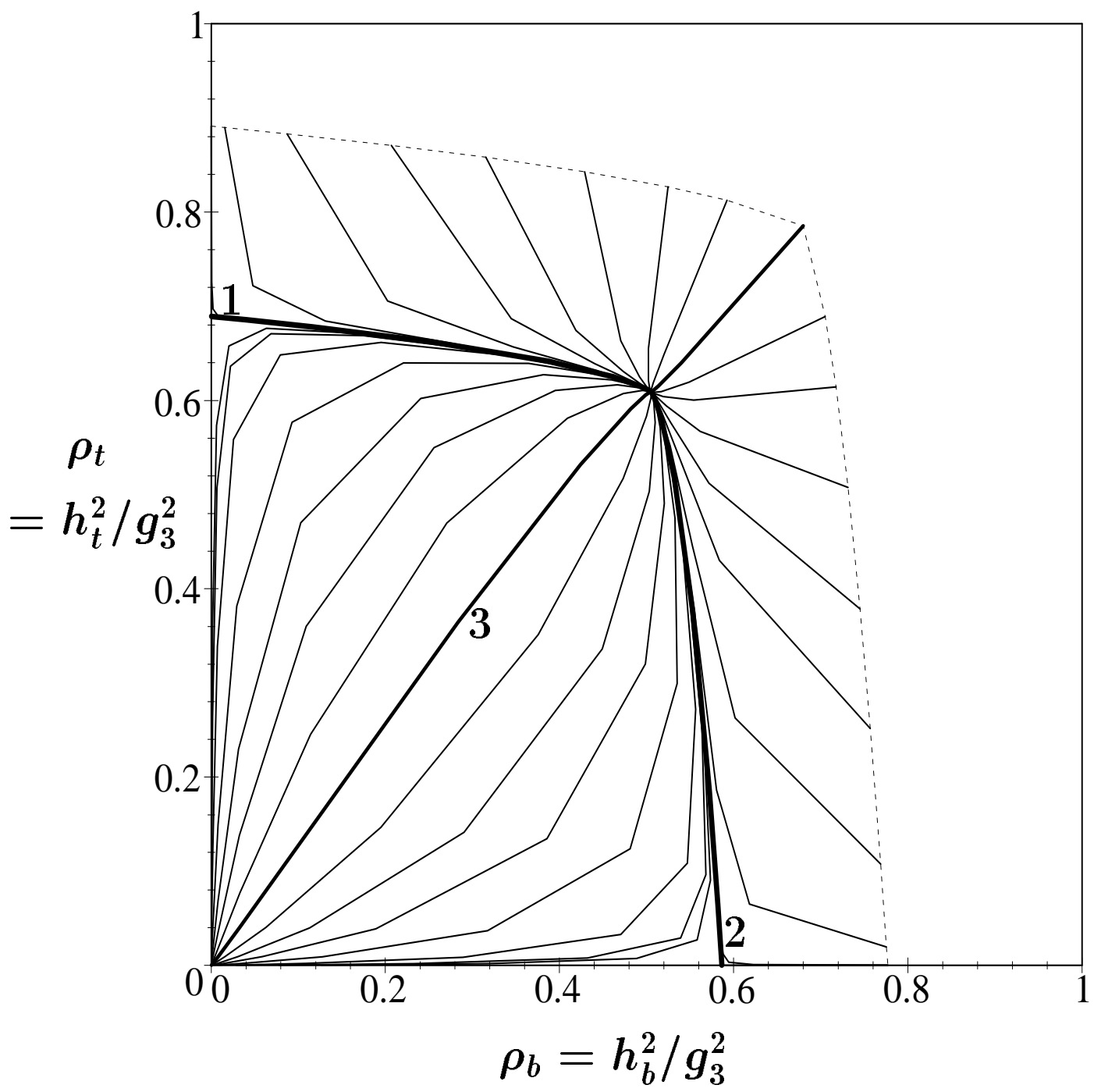

Figure 1: The two-loop renormalization group flow from $\mu=M_{G U T}$ to $\mu=174 \mathrm{GeV}$ in the $\rho_{t}$ $-\rho_{b}$ plane: first towards the more IR attractive fixed line with the two branches, labelled by "1" and "2", and then practically along it towards the IR fixed point at $\rho_{t}=.609, \rho_{b}=.505$. The less IR attractive fixed line, $\rho_{t} \simeq 1.2 \rho_{b}$, labelled by " 3 ", as well as the IR fixed point automatically imply approximate top-bottom Yukawa coupling unification. The dashed curve is the triviality bound. 


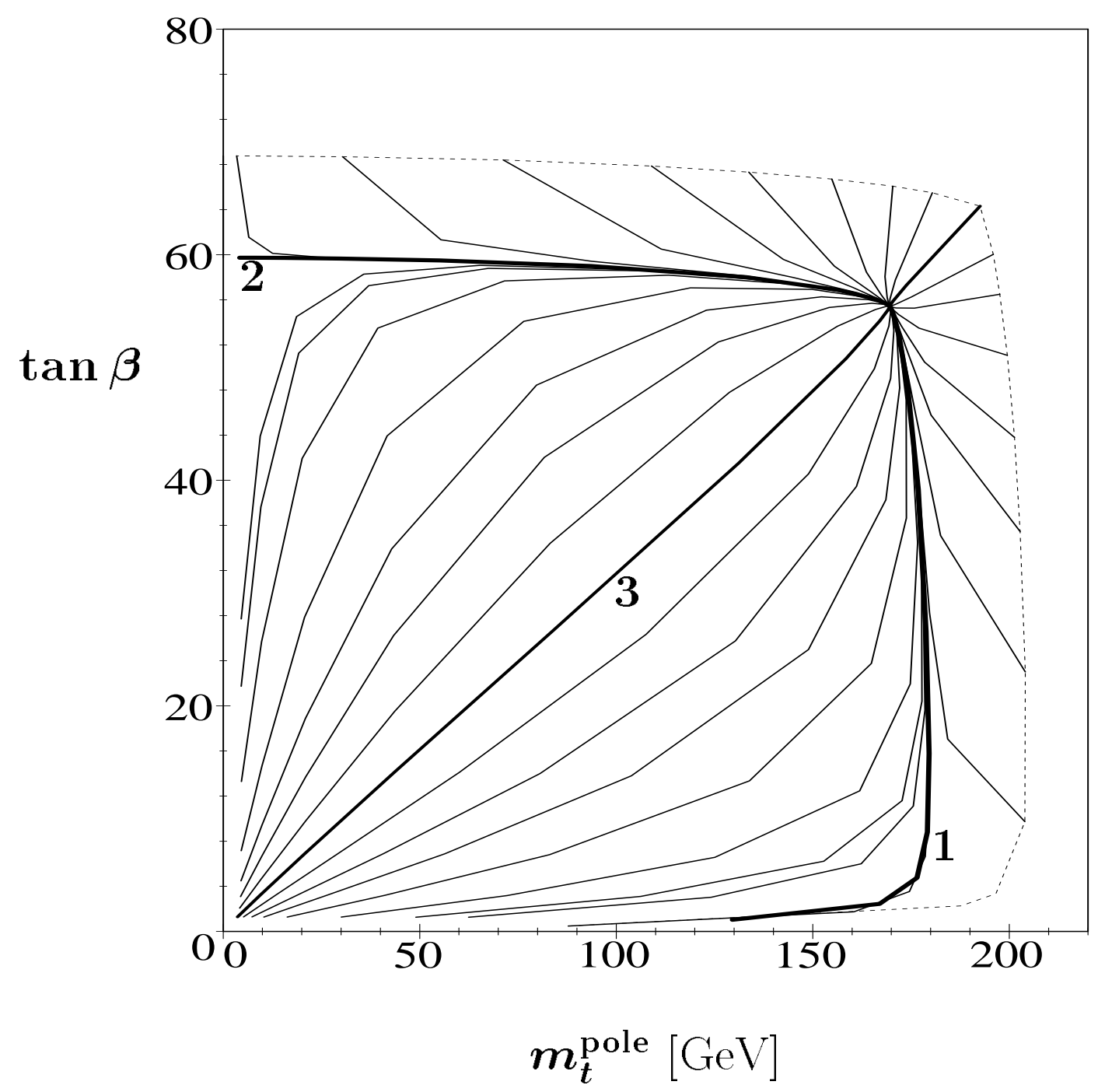

Figure 2: The two-loop renormalization group flow from $\mu=M_{G U T}$ to $\mu=174 \mathrm{GeV}$ in the $\tan \beta-m_{t}$ pole plane, as obtained from Fig. 1 for $m_{b}=4.25 \mathrm{GeV}$ : first towards the IR attractive fixed line, with the two branches $m_{t}^{\text {pole }} \simeq(170-180) \mathrm{GeV} \sin \beta$ labelled by "1", and $\tan \beta \simeq$ $0.55-0.60$, labelled by " 2 ", and then practically along it towards the IR fixed point at $m_{t}^{\text {pole }}=$ $170 \mathrm{GeV}, \tan \beta=55$. Of physical interest is the branch " 1 " including the fixed point, which imply an almost constant top mass $m_{t}^{\text {pole }} \simeq 168-180 \mathrm{GeV}$ for $2.5 \lesssim \tan \beta \lesssim 55$; along this branch approximate tau-bottom Yukawa coupling unification is realized at $M_{\mathrm{GUT}}$. The less attractive IR fixed line $m_{t}^{\text {pole }} \simeq 3 \mathrm{GeV} \tan \beta$, labelled by "3", as well as the IR fixed point automatically imply approximate top-bottom Yukawa coupling unification at all scales $\mu$. The dashed curve is the triviality bound; one of its branches corresponds to the well-known effective IR fixed point $m_{t}^{\text {pole }}=O(200 \mathrm{GeV}) \sin \beta$. 


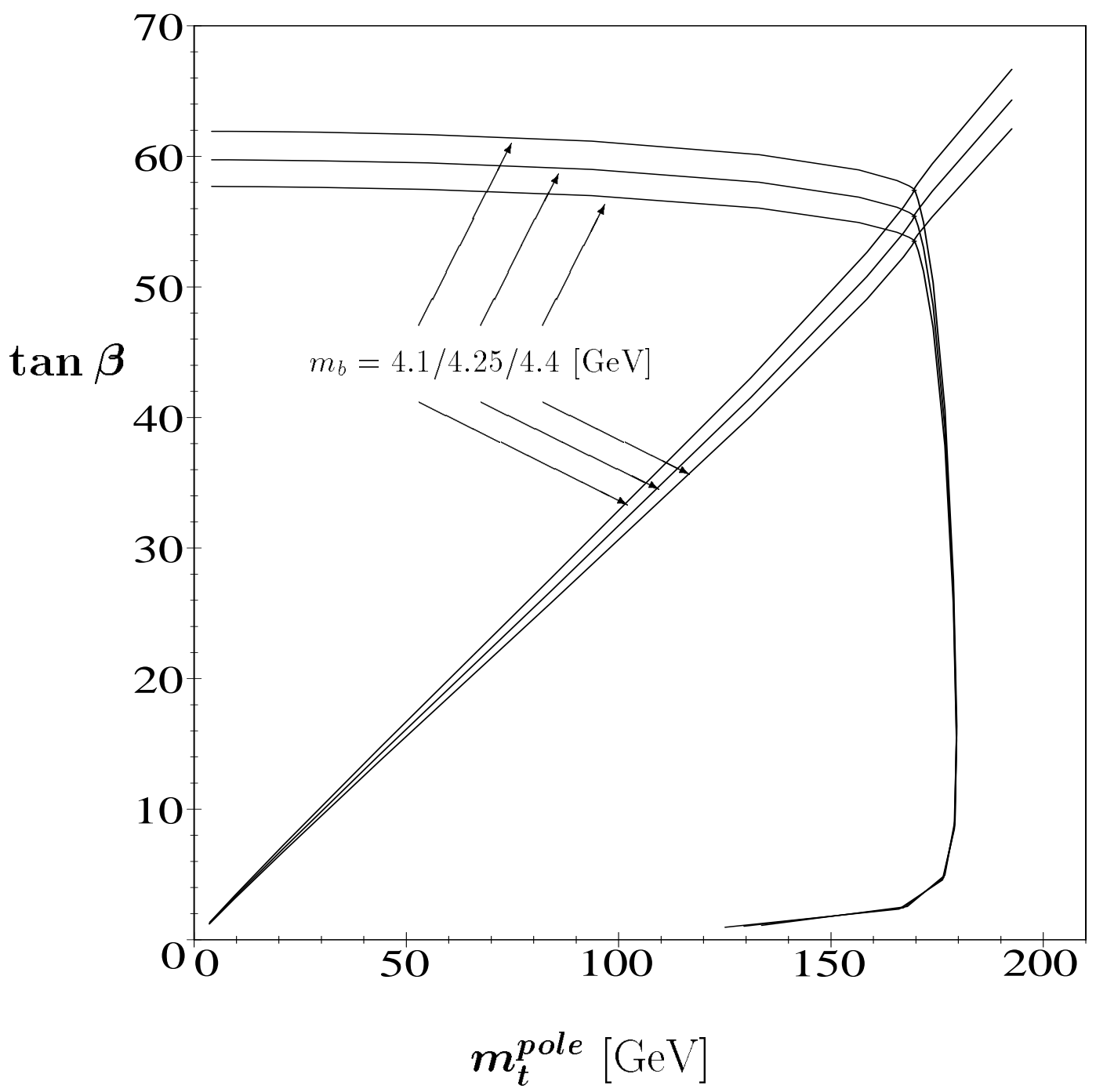

Figure 3: The more IR attractive fixed lines (square type) and the less IR attractive fixed lines (straight line type) for $m_{b}=4.1,4.25,4.4 \mathrm{GeV}$, intersecting in their respective IR fixed points $\tan \beta=57.4,55.4,53.5, m_{t}^{\text {pole }}=170 \mathrm{GeV}$. The less IR attractive fixed line as well as the IR fixed point automatically imply approximate top-bottom Yukawa coupling unification. 